UDC: 811.134.2:811.163.41'373.2

DOI: https://doi.org/10.18485/hispserb.2019.2.ch6

\author{
César Luis Díez Plaza ${ }^{1}$ \\ Instituto Cervantes, Belgrado \\ Serbia
}

\title{
LA TRANSLITERACIÓN COMO UNA OPERACIÓN: EL CASO DE <CEPBAHTEC >
}

\begin{abstract}
Resumen
La adaptación de nombres de una lengua a otra es un objeto de estudio de la Teoría de la Notación (TN). En esta teoría, se identifican la dimensión gráfica de los textos $(<\mathrm{T}>)$ y la de la notación científica $(<\mathrm{NC}>)$, relacionándolas a través de dos operaciones: la transcripción y la transliteración. La definición de esta última, entendida como una relación entre los elementos de dos conjuntos que produce una serie de pares denominados caracteres, es uno de los objetivos de este trabajo. El ejemplo elegido para explicar los conceptos teóricos es la cadena de grafemas pertenecientes al alfabeto cirílico $<$ Сервантес $>$ у su relación con las cadenas $<$ Servantes $>$ y $<$ Cervantes $>$. La conclusión final es que la notación de los textos científicos que hablan sobre problemas gráficos es un objeto de estudio por sí mismo.
\end{abstract}

Palabras clave: carácter, glifo, grafema, transcripción, transliteración.

\footnotetext{
${ }^{1}$ cdplaza@cervantes.es
} 


\section{Dando un nombre a la cuestión}

El apellido Cervantes, escrito aquí en la forma ortográfica del castellano actual (usando para ello una fuente Times New Roman, tamaño 12), se puede escribir de muchas otras formas usando tradiciones gráficas diferentes. Por ejemplo, en la página de Wikipedia dedicada a Miguel de Cervantes Saavedra en hebreo se encuentra la forma סעטנאֵוורעם.

El interés por el estudio de las diferentes formas gráficas que puedan encontrarse para notar este caso concreto (o cualquier otro nombre propio) es lo que motiva este trabajo que parte de una serie de premisas iniciales:

1. El conjunto formado por todas las formas gráficas utilizadas para notar el apellido Cervantes constituye un objeto de estudio por sí mismo.

2. Dicho objeto entraría dentro del campo de estudio de una teoría denominada la teoría de la notación y abreviada como TN.

3. También entrarían en el campo de estudio de la TN la relación entre las posibles formas gráficas que puedan generarse y la conversión de éstas en formas "fónicas". Por esta razón, la TN se relaciona con partes de la lingüística como son la fonología y la fonética y con las tradiciones culturales que se identifican como la ortografía de los sistemas gráficos adaptados para las diferentes lenguas. Queda fuera del alcance del estudio de este trabajo la reflexión sobre si la ortografía, entendida como competencia (y unida a la competencia denominada ortoépica), cae dentro del campo de la competencia lingüística (como defiende el MCER) o no.

\section{Primer paso: la dimensión gráfica de los textos}

Dentro de la TN se identifica una primera dimensión que es la dimensión gráfica de los textos y que estaría formada por cualquier texto escrito en cualquiera de los sistemas de escritura usados a través de la historia de la escritura - logosilabográfico, silabográfico, abjad, abugida, alfabético o de rasgos. Esta primera dimensión se abrevia por medio del símbolo < $>$ y la unidad mínima de la misma se denomina grafema. Se considerarían grafemas tanto los logogramas (usados en la escritura china), como los silabogramas (de la escritura japonesas) o las letras de cualquier alfabeto. La convención propuesta para representar cualquier grafema es utilizar corchetes angulares, $<>$, y las fuentes originales del sistema gráfico que se cite. 
En nuestro caso de estudio la $<\mathrm{T}>$ estaría formada por cualquier cadena de grafemas que quisiera representar la palabra Cervantes. La Tabla 1 muestra ejemplos en seis lenguas notadas con cada uno de los seis posibles sistemas de escritura identificados. El método para encontrar estas cadenas de grafemas ha sido buscar en las páginas de Wikipedia dedicadas a Cervantes en cada una de las lenguas.

\begin{tabular}{|c|c|c|c|}
\hline LENGUA & SISTEMA & EJEMPLOS & UNIDADES \\
\hline $\begin{array}{c}\text { Chino } \\
\text { (simplificado) }\end{array}$ & logosilabográfico & 寨透提断 & logograma \\
\hline Japonés & silabográfico & セルバンテス & silabograma \\
\hline Arabe & abjad & سرفاقتس & letra \\
\hline Hindi & abugida & सर्वेटिस & letra \\
\hline Español & alfabeto & Cervantes & letra \\
\hline Coreano & "featural" & 세르반테스 & letra \\
\hline
\end{tabular}

TABLA 1: Cadenas de grafemas nombrando a Cervantes en diferentes lenguas.

Como se puede observar el número de grafemas que forma cada cadena puede variar de un sistema a otro (desde los nueve que aparecen en el sistema alfabético usado para notar el español, hasta los cuatro usados en el sistema logosilabográfico del chino). Tradicionalmente a los elementos identificados como unidades de cada sistema gráfico se les ha denominado de una manera determinada - logograma, silabograma o letra. Una de las propuestas teóricas de este trabajo es unificar las denominaciones bajo un único término, el grafema, que se convierte en la unidad básica de la $\langle\mathrm{T}\rangle$. De esta manera, a un nivel teórico, resulta más fácil hablar y comparar los diferentes "objetos" que forman las cadenas de grafemas.

\section{Un caso específico: la notación gráfica en las lenguas eslavas}

Dentro del conjunto de todas las cadenas gráficas que forman la $<\mathrm{T}>$ de la palabra Cervantes, para este trabajo, interesa especialmente el subconjunto formado por las representaciones gráficas (notaciones) usadas para el grupo de las lenguas eslavas. En concreto, se han examinado las páginas de Wikipedia redactadas en once lenguas (bielorruso, búlgaro, checo, croata, eslovaco, esloveno, macedonio, polaco, rusino, ruso, serbio y ucraniano). Los datos obtenidos se reproducen en la siguiente tabla. 


\begin{tabular}{|c|c|c|c|c|}
\hline LENGUA & SISTEMA & EJEMPLOS & UNIDADES & \\
\hline ucraniano, ruso & \multirow{3}{*}{$\begin{array}{l}\text { alfabeto } \\
\text { cirílico }\end{array}$} & Серва́нтес & \multirow{5}{*}{ letra } & \\
\hline bielorruso & & Серва́нтэс & & \\
\hline $\begin{array}{l}\text { búlgaro, macedonio, } \\
\text { rusino, serbio }\end{array}$ & & Сервантес & & \\
\hline serbio & \multirow[b]{2}{*}{ alfabeto latino } & Servantes & & \\
\hline $\begin{array}{l}\text { croata, checo, esloveno, } \\
\text { eslovaco, polaco }\end{array}$ & & Cervantes & & \\
\hline
\end{tabular}

TABLA 2: Cadenas de grafemas nombrando a Cervantes en lenguas eslavas.

Lo común a las once lenguas es que todas usan como sistema gráfico el alfabético, por lo que en este caso sería posible usar el término letra como sinónimo de grafema; aunque desde un punto de vista teórico sea más adecuado el uso del segundo. La principal diferencia que separa a las lenguas citadas en dos grupos es el alfabeto utilizado, el cirílico o el latino (ambos, en todo caso, descendientes de variedades del alfabeto griego). Un caso especial, que será analizado posteriormente, es el del serbio ya que admite el uso de los dos alfabetos.

Dentro de las siete lenguas que usan el alfabeto cirílico se pueden establecer dos diferencias. La primera de ellas es la aparición de un único grafema diferente en la cadena de nueve grafemas: el grafema $<\boldsymbol{\text { }}>$ del bielorruso, frente al grafema $<\mathrm{e}>$ empelado en ucraniano y ruso. La segunda diferencia es la aparición de una tilde sobre el grafema $a$ en bielorruso, ruso y ucraniano, <á>. Desde el punto de vista de la TN, el uso de la tilde en estos casos lleva a formularse la pregunta de qué tipo de representación son dichas cadenas de grafemas, ya que - por ejemplo - para el ruso no podría tratarse de una forma ortográfica ya que la ortografía rusa no emplea la tilde, a no ser que se trate de textos dedicados al aprendizaje de la lengua rusa por parte de extranjeros.

Cuando se habla de las lenguas que usan el alfabeto latino, es necesario explicar la diferencia entre los dos tipos de cadenas que aparecen: <Cervantes $>v s$. <Servantes $>$. En el primer caso (que aparece en croata, checo, esloveno, eslovaco y polaco), desde un punto de vista gráfico lo que se tiene es una cadena idéntica a la que aparece en la lengua original, el español. Se están usando los mismos grafemas, por lo que no se hace ninguna adaptación del nombre. Un asunto diferente es cómo leerán estás cadenas los hablantes nativos (y alfabetizados) de estas lenguas. Es 
muy posible que la "pronunciación" de dichas cadenas sea muy diferente de una lengua a otra (e, incluso, entre dialectos de una misma lengua). En el caso de la segunda cadena, $<$ Servantes $>$ (empleada en serbio), estamos ante una situación diferente ya que sí se produce una adaptación del nombre, el primer grafema no es igual en la cadena gráfica serbia y en la original española. Es necesario dar una explicación a este hecho.

Indagar en estas cuestiones y comprender mejor los procesos que han intervenido en la adaptación de la representación gráfica de un nombre por parte de una tradición gráfica diferente es uno de los objetos principales de la TN. En dichos procesos, aparecen diferentes operaciones, como es el caso de la transliteración. En el siguiente apartado, se tratará con detenimiento el caso mencionado, las formas gráficas adoptadas para notar la lengua serbia, ya que en ésta la operación de la transcripción es especialmente relevante.

\title{
4. El caso del serbio: introducción a la operación de transliteración
}

La Constitución de la República de Serbia de 2006, en su artículo 10, habla de los usos de lengua y escrituras en el país.

\author{
Језик и писмо \\ Члан 10.
}

У Републици Србији у службеној употреби су српски језик и ћириличко писмо.

Службена употреба других језика и писама уређује се законом, на основу Устава.

Устав Републике Србије, 2006

Lengua y escritura

Artículo 10

En la República de Serbia tienen un uso oficial la lengua serbia y el alfabeto cirílico.

El uso oficial de otras lenguas y alfabetos se regulará por ley sobre lo establecido por la Constitución.

Constitución de la República de Serbia, 2006 
En este artículo no sólo se establece el uso oficial de una lengua, el serbio, sino que además se incluye la "escritura" oficial de la misma. Aunque, al igual que ocurre con las lenguas, se reconoce el uso de otras escrituras regulado por las leyes. Dicho uso es una realidad en el país que puede constatarse al pasear por cualquier ciudad y ver como se alternan los letreros en cirílico o latino, o como hay prensa que se edita sistemáticamente en el primero de los alfabetos (el periódico Политика o el semanario $H И H$ ) y otra que lo hace en el segundo (Danas y Vreme por poner también un ejemplo de un periódico y un semanario). En el sistema educativo, los niños aprenden ambos sistemas (empezando por el cirílico), y más tarde los ciudadanos pueden usar cualquiera de ellos, aunque en la actualidad parece que existe un mayor uso del alfabeto latino (las comunicaciones usando soportes digitales - teléfonos, ordenadores, etc. - han podido influir en esta tendencia).

Desde un punto de vista general, se podría decir que la relación entre las cadenas de grafemas $<$ Servantes $>$ y $<$ Сервантес $>$ es el resultado de aplicar la operación de la transliteración sobre cualquiera de ellas. El número de grafemas no varía, nueve en total, y algunos de ellos (los utilizados para notar las vocales $a$ y $e$ ) son comunes a ambos sistemas gráficos. Ahora bien, desde el punto de vista de la TN es necesario definir qué se entiende por la operación de transliteración, abreviada TRaL.

En la bibliografía se pueden encontrar definiciones de transliteración como las siguientes (ordenas por criterio cronológico, de aparición de la obra).

\begin{tabular}{|l|l|l|}
\hline LÁZARO & $1968^{3}$ & $\begin{array}{l}\text { Transliteración. } \\
\text { Transcripción de las palabras escritas en un } \\
\text { alfabeto, con letras de otro alfabeto más familiar } \\
\text { al lector. La transliteración se efectúa letra a letra, } \\
\text { según correspondencia de los sonidos por ellas } \\
\text { representados. Cuando dicha correspondencia no es } \\
\text { exacta suelen emplearse diacríticos auxiliares. Vid. } \\
\text { Transcripción. }\end{array}$ \\
\hline 1978 & $\begin{array}{l}\text { Bibliographic transliteration is the operation of } \\
\text { converting the characters of a source script into the } \\
\text { character of a target script. In principle, this is a one- } \\
\text { to-one transformation, in which one character of the } \\
\text { source script is converted into one (and only one) } \\
\text { specific character of the target script. }\end{array}$ \\
\hline
\end{tabular}




\begin{tabular}{|l|l|l|}
\hline DRAE & $2001^{22}$ & $\begin{array}{l}\text { transliterar. } \\
\text { (De trans-y el lat. littlěra, letra). tr. } \\
\text { Representar los signos de un sistema de escritura } \\
\text { mediante los signos de otro. }\end{array}$ \\
\hline Mª MOLINER & $2007^{3}$ & $\begin{array}{l}\text { transliteración } \mathrm{f} . \\
\text { Representación de los sonidos de una lengua con los } \\
\text { signos alfabéticos de otra. }\end{array}$ \\
\hline
\end{tabular}

La primera definición corresponde al Diccionario de términos filológicos de Lázaro Carretery en ella aparecen los rasgos más importantes de la operación: la existencia de dos alfabetos y el establecimiento de una relación entre las "letras" de uno y las del otro. También se indica que "la transliteración se efectúa letra a letra, según la correspondencia de los sonidos por ellas representados". El problema que se percibe en esta definición es que parece que sólo se podría transliterar de un sistema alfabético a otro también alfabético, dejando sin tratar las transliteraciones entre sistemas diferentes como, por ejemplo, de un sistema silabográfico a uno alfabético o viceversa.

La segunda definición viene de una monumental obra dedicada al estudio de la conversión de las escrituras desde un punto de vista concreto, el de las necesidades de los profesionales de la biblioteconomía y la documentación. Por esta razón, el adjetivo "bibliographic" acompaña al sustantivo "transliteration". Independientemente de esta aclaración sobre su origen, la definición propuesta por Wellisch es la mejor ya que incluye todos los elementos que intervienen en la operación: dos sistemas gráficos, el de la lengua fuente y el de la lengua meta, y la relación entre los elementos de ambos sistemas. Esta relación convierte a un elemento del primer sistema en un elemento (y sólo en uno) del segundo sistema.

La tercera, perteneciente al Diccionario de la Real Academia, es una formulación abreviada de lo dicho hasta ahora (pensada para un público general y no especialista) y funciona bastante bien si se entiende la palabra "signo" de una manera muy general. Utilizar esta palabra de esa manera, por ejemplo, elimina el problema que aparecía en la definición de Lázaro Carreter al usar un término tan específico como el de "letra".

La última definición, extraída del estupendo Diccionario de María Moliner, implica un problema ya que mezcla dos dimensiones diferentes: la gráfica y la fónica ("[r]epresentación de los sonidos de una lengua con los signos alfabéticos de otra”); y la transliteración sólo opera 
relacionando cadenas pertenecientes a una de ellas (la gráfica). Cuando se relacionan ambas dimensiones estamos ante una operación diferente, la transcripción (abreviada TRaC).

Sobre la base de lo expuesto se puede definir la transliteración, $T R a L$, como una operación que convierte una cadena de grafemas de un determinado sistema gráfico (el de la lengua origen) en otra de un sistema diferente (el de la lengua meta) estableciendo una relación de uno a uno entre los grafemas de ambos sistemas. Si usáramos el lenguaje de la teoría de conjuntos podríamos decir que se está estableciendo una relación entre los elementos de dos conjuntos (el conjunto origen y el conjunto meta). Lo ideal, además, es que está relación fuese biyectiva; es decir que todos los elementos del conjunto origen tuvieran una imagen distinta en el conjunto meta, y que a cada elemento del conjunto meta le correspondiera un elemento del conjunto origen.

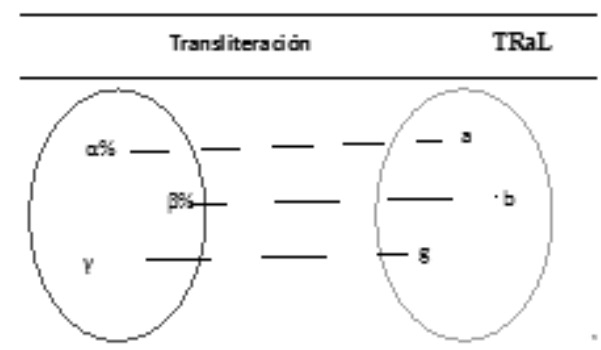

ESQUEMA 1: La operación de la transliteración.

El ceñirse a la etimología del término transliteración (que explica, por ejemplo, el DRAE "de trans- y el lat[ín] littlěra, letra") hace pensar que esta operación sólo se puede establecer entre sistemas de escritura basados en "letras", algo que no es cierto. Por ejemplo, veamos el siguiente ejemplo de TRaL:

Definamos los dos conjuntos:

Conjunto origen: versión del alfabeto latino usada en la notación gráfica del español (27 elementos)

Conjunto meta: subconjunto del conjunto de los números naturales formados por el intervalo cerrado $[1,27]$ y representado por el sistema gráfico posicional que usa diez cifras: " $0,1,2,3,4,5,6,7$, 8 y 9". 
Es importante señalar que ambos conjuntos poseen un orden en sus elementos (están ordenados). En el caso del conjunto meta, el orden lo marcan las relaciones "ser mayor o menor que" y se podría definir como algo intuitivo (alguien sabe ordenar dicho conjunto, aunque no sepa exactamente cómo lo hace). El resultado de la ordenación sería: 1, 2, 3... 27.

El conjunto origen, la versión del alfabeto latino usada en la notación gráfica del español, también está ordenado, aunque esto no es un hecho intuitivo, sino cultural. Al aprendiz (niño o adulto) se le enseña el orden del alfabeto para que, por ejemplo, pueda buscar las palabras en un diccionario: "A, B, C... Z".

Usando precisamente esa característica de "conjuntos ordenados" se puede aplicar la operación de la $T R a L$ en este caso, asignando a cada grafema del conjunto origen (una letra) un grafema del conjunto meta (un número) según la posición que la letra ocupe en el orden del alfabeto. De esta manera se crearán 27 pares ordenados como los siguientes: $\langle A: 1\rangle$, $<\mathrm{B}: 2>\ldots<\mathrm{Z}: 27>$. Desde la TN defendida en este trabajo a cada uno de esos pares ordenados se le denominará carácter. El carácter es la unidad mínima de la $T R a L$, de la misma manera que el grafema era la unidad mínima de la dimensión gráfica de los textos, $<\mathrm{T}>$.

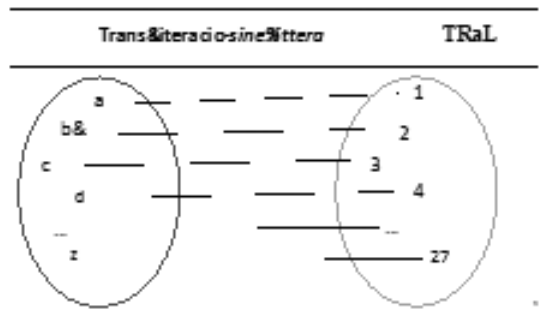

ESQUEMA 2: Transliteración a un "lenguaje" no alfabético.

Teniendo todo esto presente una primera propuesta para transliterar la cadena <cervantes> sería la siguiente:

1) $<$ cervantes $>\rightarrow<35192311421520>$

Ahora bien, el problema con la cadena resultante es que tiene más elementos que la anterior (desde un punto de vista gráfico) y que al hacer la operación inversa no aparece el resultado esperado, sino uno diferente (como se ve en 2): 
2) $<35192311421520>\rightarrow<$ ceaibcadbaes $>$

Una solución a este problema es intercalar un "signo" auxiliar (un punto) entre los elementos del conjunto meta para asegurar la correcta relación entre estos y los del conjunto origen, como aparece en 3):

3) $<3.5 .19 .23 .1 .14 .21 .5 .20>\rightarrow<$ cervantes $>$

Ahora bien, el problema de la lectura de cualquiera de estas cadenas, o sea, el dotar de una estructura fónica a una representación gráfica, nos presenta la otra operación que aparece en los procesos de adaptación de sistemas gráficos y que también es necesario definir: la transcripción.

\section{Hacia una definición de transcripción}

La siguiente definición de la operación de transcripción (abreviada, $T R a C$ ) propuesta por Mosterín (1993) es muy completa y adecuada:

Transcripción fonémica: Representación gráfica biunívoca de la secuencia de fonemas proferida por un hablante. No hay que confundir la transcripción fonémica (realizada por los fonólogos con fines científicos) con la ortografía fonémica (que es el tipo ideal de ortografía para la escritura alfabética corriente de toda comunidad de hablantes). La primera no admite desviaciones del principio fonético en ningún caso; la segunda las admite siempre que ello facilite la práctica de la comunicación escrita.

Transcripción fonética: Representación biunívoca de la cadena sonora proferida por un hablante en función de su análisis en una serie de fonos establecida de antemano. La transcripción fonética (a diferencia de la fonémica) admite muchos grados distintos de finura, según que el fonetista quiera distinguir más o menos fonos en su análisis.

Lo primero que hay que señalar es que el autor usa el término "fonémico" como sinónimo de "fonológico"; por lo tanto, se hablaría de transcripción fonológica, abreviada por /TRaC/; y transcripción fonética, [TRaC]. La convención para distinguir las abreviaturas ha sido utilizar la habitual diferencia de colocarlas entre corchetes ([ ]) o barras (/ /).

Como se puede observar en la definición, Mosterín también habla en el caso de la transcripción, como ocurría en el de la transliteración, 
de representaciones (correspondencias) biunívocas, aunque esta vez se establecen entre conjuntos diferentes. Para este autor, la transcripción "fonémica" establecería una relación entre los fonemas "preferidos" por un hablante y los elementos de la representación gráfica que se adopte para ello; mientras que la transcripción fonética haría lo mismo entre "fonos" (sonidos) y los elementos de la representación gráfica. Aunque podamos estar casi de acuerdo en todo con Mosterín (salvando detalles de terminología), hay que señalar una crítica: "los fonemas" no los profiere el hablante.

En todo caso, el esquema de la operación de $T R a C$ sería el siguiente:

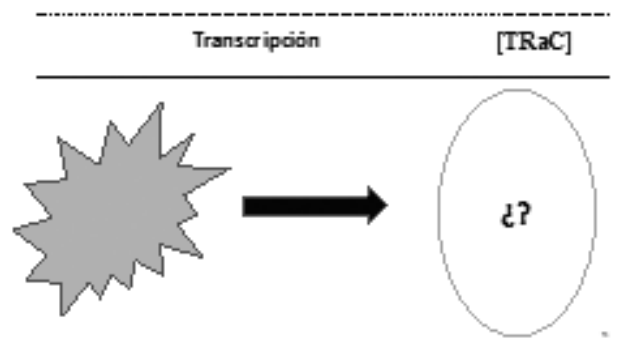

ESQUEMA 3: Esquema de la operación transcripción

A la izquierda del esquema, la nube estrellada intenta representar el mundo del sonido, el mundo fónico, el de aquello que se produce y se recibe. Si ese mundo se analiza con una gran variedad de detalles articulatorios y acústicos estaríamos ante un caso de análisis fonético; si, por el contrario, lo que interesa es el grupo de operaciones mentales que permiten a los hablantes identificar las cadenas de sonidos representativas estaríamos hablando de un análisis fonológico. En todo caso, lo importante es que se trata de una relación vertical entre dos planos diferentes: el plano fónico y el plano gráfico. Algo que no ocurría en el caso de la transliteración donde se establecía una relación horizontal, en el mismo plano: el plano gráfico.

A la derecha del esquema, encontramos un diagrama de Venn con signos de interrogación. Esta representación quiere decir que es necesario definir un conjunto de elementos que sirva de conjunto meta en la relación establecida. Ese conjunto de elementos formará el sistema de transcripción elegido. Los sistemas de transcripción pueden ser de 
diversos tipos: sistemas "alfabéticos", como el de la Revista Española de Filología o el de la Asociación Fonética internacional (IPA por sus siglas en inglés); o sistemas "no alfabéticos" como el Visible speech, de Bell o la transcripción icónica de Jespersen (estos últimos han tenido una acogida mucho menor entre la comunidad científica). En todo caso, lo que hace la $T R a C$ es, una vez identificados los elementos del conjunto origen - es decir, después de hacer discreta la dimensión fónica (la cadena hablada), de por sí continua - representarlos por medio de un sistema gráfico para que un lector los pueda identificar.

Veamos este proceso con un ejemplo. La cadena de grafemas $<$ cervantes > del español puede ser analizada como las siguientes cadenas de símbolos alfabéticos:

\begin{tabular}{|c|c|}
\hline Transcripciónfonética & TRaC \\
\hline 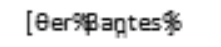 & IPA \\
\hline [ & RFE \\
\hline
\end{tabular}

TABLA 3: Transcripciones propuestas para la cadena <Cervantes> en español.

En la primera cadena, aparecen los signos del alfabeto de IPA; mientras que en la segunda aparecen los del alfabeto de la Revista de Filología Española. Las diferencias son mínimas, pero existen. Al tratarse de signos que representan sonidos (se usan los corchetes) estamos ante una transcripción fonética, [TRaC].

Un caso diferente sería el de las transcripciones propuestas para la cadena <cervantes> en el caso del inglés:

\begin{tabular}{cc}
\hline Transcripción fonológica & TRaL $/$ \\
\hline /sar'vænti:z/ & IPA - UK \\
/sar'va:nterz/ & IPA - USA
\end{tabular}

TABLA 4: Transcripciones propuestas para la cadena $<$ Cervantes > en inglés. 
Aquí se está intentando representar dos variedades dialectales del inglés (la británica frente a la estadounidense) utilizando para ambos casos el alfabeto de la IPA. Curiosamente ambas representaciones quieren capturar la imagen mental (fonológica) que un hablante nativo inglés asociaría a la cadena de grafemas <cervantes $>$. En las /TRaC/ es necesario citar la lengua que se está trascribiendo ya que un determinado signo puede ser un fonema en una lengua y no serlo en otra. Por ejemplo, /v/ no existe como fonema en ningún dialecto del español y sí aparece en los dialectos del inglés.

Todo lo expuesto hasta el momento se debe relacionar dentro del modelo propuesto por la teoría de la notación, TN, anteriormente presentada.

\section{Modelo de la teoría de la notación (TN)}

La TN pretende investigar las relaciones existentes entre dos dimensiones diferentes: la dimensión gráfica de los textos, $\langle\mathrm{T}\rangle$, y la dimensión gráfica de la notación científica, $<\mathrm{NC}>$. La primera es la presentada al principio de este trabajo y un ejemplo de la segunda es este propio trabajo. Cuando reflexionamos por escrito sobre un tema de notación o escritura estamos creando un texto científico que se puede entender como un objeto de estudio en sí mismo.

Estas dos dimensiones se relacionan por medio de dos operaciones diferentes: la transliteración, TRaL, y la transcripción. Esta última, además, se podría subdividir en dos: transcripción fonética [TRaC] y fonológica / $\mathrm{TRaC} /$.

Para cada una de las dos dimensiones y de las dos operaciones se definen unas unidades específicas: grafemas, glifos, caracteres y símbolos.

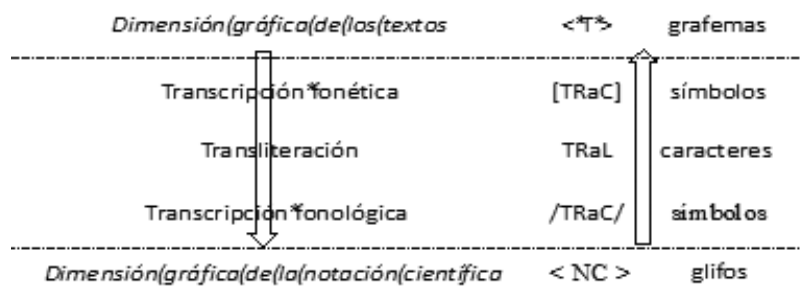

TABLA 5: Modelo propuesto de la Teoría de la Notación. 
Hasta ahora, en este trabajo, se han presentado tres unidades grafemas, caracteres y símbolos; sólo es necesario explicar la cuarta: el glifo. Un glifo aparece cuando, en un texto científico, se habla de cualquier grafema, carácter o símbolo. Eso quedará más claro cuando se desarrolle completamente el caso de <cervantes $>$. Pero antes de llegar a ese caso es necesario presentar una propiedad más del modelo: la propiedad "reflexiva".

Anteriormente, se ha dicho que el modelo relaciona dos dimensiones, la $\langle\mathrm{T}>$ y la $\langle\mathrm{NC}>$; aunque también se podrían estudiar relaciones que se establecieran entre representantes de la misma dimensión. Por ejemplo, si hablamos del caso estudiado, <Cervantes Сервантес> estamos hablando de la relación que se establece entre dos $<\mathrm{T}>$; y, si tratamos la diferencia entre el signo $\hbar$ usado por el alfabeto de la RFE y el signo $\beta$ usado por la AFI, estamos analizando la relación establecida entre dos $<\mathrm{NC}>$.

\section{El modelo ejemplificado en el caso de la cadena Cervantes}

Como ya se ha dicho, el objetivo es analizar la relación que se puede establecer entre las cadenas $<$ Cervantes $>$ y $<$ Сервантес $>$. Dicha relación aparece en el siguiente esquema:

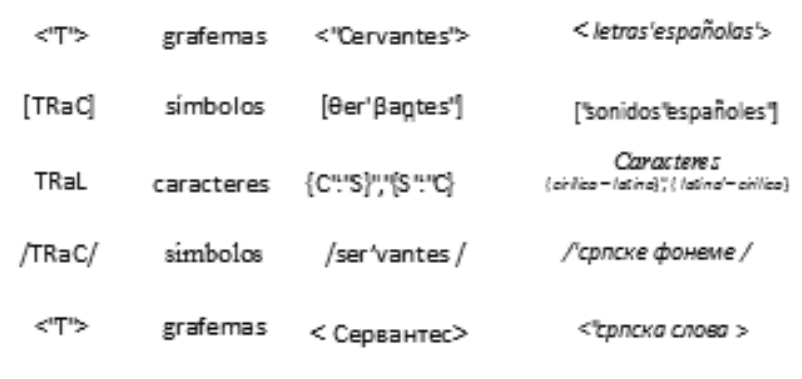

TABLA 5: El modelo propuesto para el caso de las cadenas $<$ Cervantes $>y$ $<$ Сервантес>.

Como ambas cadenas aparecen en los sistemas de notación empleados en español (alfabeto latino) y en serbio (alfabeto cirílico en este caso), se puede decir que son dos cadenas de nueve grafemas. Cada 
una de estas cadenas de grafemas puede ser analizada por medio de la transcripción (TRaC) y generar distintas cadenas de signos fonéticos o fonológicos. En estos casos, hay que tener en cuenta que se toman diferentes decisiones científicas. Por ejemplo, el grafema $<c>$ del español puede recubrir dos fonemas /s/ y / $/$ / dependiendo de dialectos y posiciones: $<\mathrm{ce}>=/ \mathrm{se} /$ en dialectos "seseantes" y $\langle\mathrm{ce}>=/ \Theta /$ en dialectos no seseantes (como el castellano septentrional). En el primer caso, las cadenas de grafemas $<$ ce $>\mathrm{y}<$ se $>$ recubrirían la misma sustancia fónica; algo que no ocurriría en los otros dialectos $/ \mathrm{se} / \neq /$ e $/$.

¿Se podría decir, entonces, que el serbio ha tomado como modelo los dialectos seseantes para hacer las adaptaciones de palabras que incorporaran grafemas como <ce>? Hacer una aseveración como esta resulta demasiado arriesgado y generalista; pero, permite iluminar áreas que deben ser investigadas. La principal de ellas afecta a la operación de transliteración, TRaL. Desde el punto de vista expuesto se define muy bien la operación como la relación entre los dos conjuntos (origen y meta); pero, no se ha explicado cómo se establece la relación, con qué criterios un elemento del primer conjunto se relaciona con uno (y sólo uno) del segundo. En el caso que servía de ejemplo antes, la TRaL desde un sistema gráfico alfabético al sistema gráfico de los "números" (<3.5.19.23.1.14.21.5.20> $\rightarrow<$ cervantes $>$ ), el principio estaba claro, se trataba del principio de ordenación: todo el mundo conoce el orden de los números naturales y el de las letras del abecedario. Pero, ¿qué ocurre en los otros casos?, ¿cómo se establece la relación entre los caracteres $<\mathrm{C}$ :s $>$ de los alfabetos cirílico y latino, o entre los caracteres $<\beta: \sigma>$ de los alfabetos griego y cirílico?, ¿sencillamente se establecen las relaciones por su posición en dichos alfabetos, criterio de ordenación? Con toda seguridad la respuesta es no, por lo que es necesario seguir buscando respuestas a preguntas como ésta. Y ese proceso lleva a plantearse las relaciones entre las dos operaciones - la $T R a L$ y la $T R a C$ - ya que aparece que el uso de "alfabetos" similares (o iguales) para ambas ha podido generar en la producción científica una confusión entre las mismas. Para separar esto es necesario volver a una propiedad del modelo mencionada anteriormente: la propiedad reflexiva que, en el caso estudiado, se podría expresar con el siguiente esquema. 


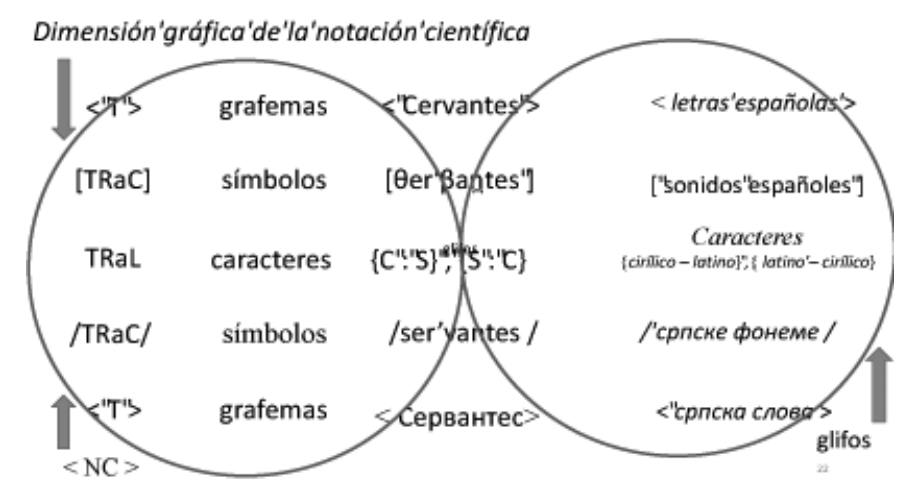

ESQUEMA 4: La propiedad reflexiva en el modelo propuesto.

Lo que pretende transmitir el esquema es que, al generar la dimensión gráfica de la notación científica, la $\langle\mathrm{NC}\rangle$, estamos creando un marco en el cual el análisis de la relación entre los elementos del mismo, los glifos, nos permita entender cómo se han llegado a constituir como tales glifos desde elementos diferentes - grafemas, símbolos o caracteres - resultado de aplicar unas operaciones, la TRaL o la TRaC, sobre una dimensión diferente, la de los textos, $<\mathrm{T}\rangle$. 0 , en nuestro ejemplo, contemplar las tres cadenas $<$ Cervantes - Servantes - Сервантес $>$ como cadenas de nueve glifos, nos permite indagar en la relación entre los grafemas $\langle\mathrm{v}>,<\mathrm{B}>$, o la que existe entre el carácter $<\mathrm{v}$ : $\mathrm{B}>$ y el signo $/ \mathrm{v} /$. Es decir, en el caso del serbio <Servantes:Сервантес> estamos ante un caso claro de transliteración, TRaL. Ahora bien, desde un punto de vista histórico, ¿cómo se estableció la relación entre los alfabetos latino y cirílico? Una pregunta que no tiene una respuesta fácil.

Como tampoco tiene una respuesta nada fácil la siguiente cuestión: ¿cuál es la relación entre la cadena española <Cervantes $>$ y la serbia <Servantes $>$ ? En este caso, ya que no parece procedente hablar de una TRaL, ¿estaremos entonces ante un caso de TRaC o ante algo diferente? Contestar a estas preguntas es el objetivo de la TN, una teoría que se podría resumir de la siguiente manera. 


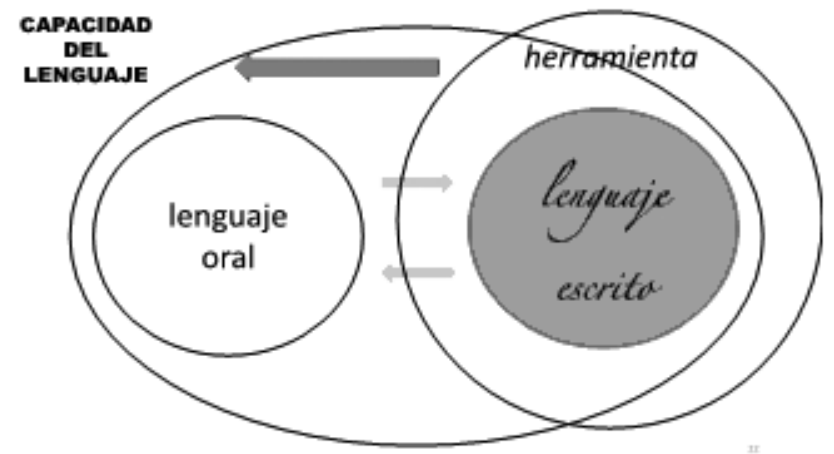

ESQUEMA 5: Resumen de la TN

La TN defiende que la capacidad del lenguaje está compuesta por dos esferas independientes, el lenguaje oral y el lenguaje escrito, entre las que se establecen distintas relaciones: por ejemplo, la reflexión científica sobre el lenguaje oral se hace mediante el lenguaje escrito (que se convierte en una herramienta para este fin), y la mayoría de reflexiones sobre este lenguaje escrito se hace en el mismo (se convierten en metadescripciones).

\section{BIBLIOGRAFÍA}

Lázaro Carreter, Fernando. Diccionario de términos filológicos. Madrid: Gredos, 1953. Impreso.

Moliner, María. Diccionario de uso del español, 3a ed., Madrid: Gredos, 2007. Impreso.

Mosterín, Jesús. Teoría de la escritura. Barcelona: Icaria, 1993. Impreso.

RAE. Diccionario de la lengua española, 22a ed., 2001. Impreso.

Wellisch, H. Hans. The Conversion of Scripts. Its Nature, History, and Utilization. New York: John Wiley \& Sons, Inc., 1978. Print. 


\section{TRANSLITERATION AS AN OPERATION: THE CASE OF <CEPBAHTEC >}

\section{Summary}

The adaptation of proper names from one language to another is an object of the study of the Theory of Notation (TN). In this theory, the graphic dimension of the texts $(<\mathrm{T}>)$ and the dimension of the scientific notation $(<\mathrm{NC}>)$ are identified and related through two operations: transcription and transliteration. The definition of the latter, understood as a relationship between the elements of two sets produced by a series of pairs called characters, is one of the main objectives of this work. The chosen example to explain the theoretical concepts is the chain of graphemes belonging to the Cyrillic alphabet $<$ Сервантес $>$ and its relationship with the chains $<$ Servantes $>$ and $<$ Cervantes $>$. The final conclusion is that the notation of the scientific texts that talk about graphic problems is an object of studies by itself.

Keywords: character, glyph, grapheme, transcription, transliteration. 